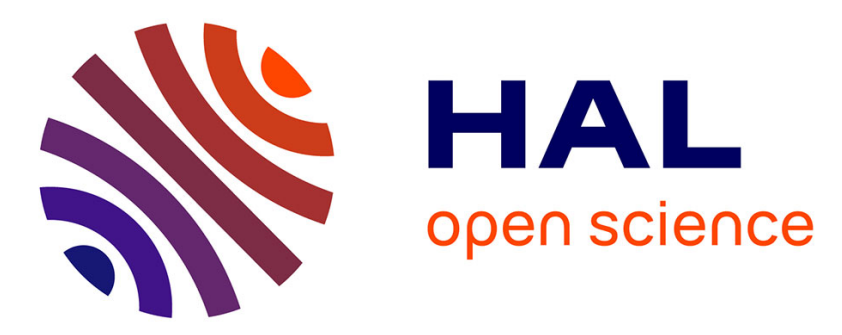

\title{
Spontaneous resistance fluctuations and their evolution near the threshold in o-TaS3 below the liquid-nitrogen temperature
}

\author{
V. Pokrovskii, S. Zaitsev-Zotov, P. Monceau, F. Nad'
}

\section{- To cite this version:}

V. Pokrovskii, S. Zaitsev-Zotov, P. Monceau, F. Nad'. Spontaneous resistance fluctuations and their evolution near the threshold in o-TaS3 below the liquid-nitrogen temperature. Journal de Physique IV Proceedings, 1993, 03 (C2), pp.C2-189-C2-192. 10.1051/jp4:1993238 • jpa-00251321

HAL Id: jpa-00251321

https://hal.science/jpa-00251321

Submitted on 1 Jan 1993

HAL is a multi-disciplinary open access archive for the deposit and dissemination of scientific research documents, whether they are published or not. The documents may come from teaching and research institutions in France or abroad, or from public or private research centers.
L'archive ouverte pluridisciplinaire HAL, est destinée au dépôt et à la diffusion de documents scientifiques de niveau recherche, publiés ou non, émanant des établissements d'enseignement et de recherche français ou étrangers, des laboratoires publics ou privés. 


\title{
Spontaneous resistance fluctuations and their evolution near the threshold in o-TaS 3 below the liquid-nitrogen temperature
}

\author{
V.Ya. POKROVSKII ${ }^{(1)}$, S.V. ZAITSEV-ZOTOV ${ }^{*}$, P. MONCEAU and F.Ya. NAD'(1)
}

Centre de Recherches sur les Très Basses Températures, CNRS, BP. 166X, 38042 Grenoble cedex, France

${ }^{*}$ Institute of Radioengineering and Electronics of RAS, Mokhovaya 11, 103907 Mosców, Russia

\begin{abstract}
It is found that below $80 \mathrm{~K} 0-\mathrm{Tas}_{3}$ demonstrates spontaneous resistance fluctuations growing when the temperature is decreasing. The average relaxation time, $\tau_{0}$, obtained from the noise spectra demonstrates activated behavior, $\tau_{0} \propto \exp (W / T), W \approx 1300 \mathrm{~K}$ for $50<T<70 \mathrm{~K}$. In the vicinity of the threshold the average frequency of the fluctuations, $f_{0}$, grows with increasing the voltage, revealing onset of the Fröhlich current. $\log \left(f_{0}\right)$ is found to be proportional to the increase of conductivity. We conclude that the main source of the resistance fluctuations are thermallyassisted jumps of dislocations of the charge-density waves.
\end{abstract}

The physical properties of quasi one-dimensional conductors with sliding charge-density waves (CDW), such as $\mathrm{TaS}_{3}, \mathrm{~K}_{0.3} \mathrm{MOO}_{3}$, below approximately half the temperature of the Peierls transition reveal a number of new features [1]: reduction of the activation energy for the low-field conductivity and disappearance of thermal hysteresis, tremendous growth of anisotropy, growth of the threshold field, $E_{T}$, for the CDW depinning and development of nonlinear conductivity at $E<E_{T^{\prime}}$ hopping conductivity. These features seem to indicate development of a new state of the CDW, probably disordered, or glassy [2]. However, the nature of the transition and the structure of the resulting state remain unclear.

In the present report we demonstrate, that below $75 \mathrm{~K}$ o-Tas exhibits spontaneous resistance fluctuations, which increase with further cooling. We show, that the fluctuations can be explained by thermally-assisted climb of dislocations, which develop in the equilibrium state of the $C D W$ at low temperature.

We performed spectral measurements of broad-band voltage fluctuations in samples of o-Tas connected in series with a source of d.c. voltage and a loading resistor. 6 samples with dimensions $(0.4 \div 1) \mathrm{mm} \times(1 \div 20) \mu \mathrm{m}^{2}$ have been studied. Both 2- and 4-contact configurations were used. The measurements were performed in the frequency range $0.2 \div 10^{3} \mathrm{~Hz}$.

RESULTS

Fig. 1 (a) presents typical dependencies of the spectral density of noise on the voltage $V$ applied to the sample at several given 


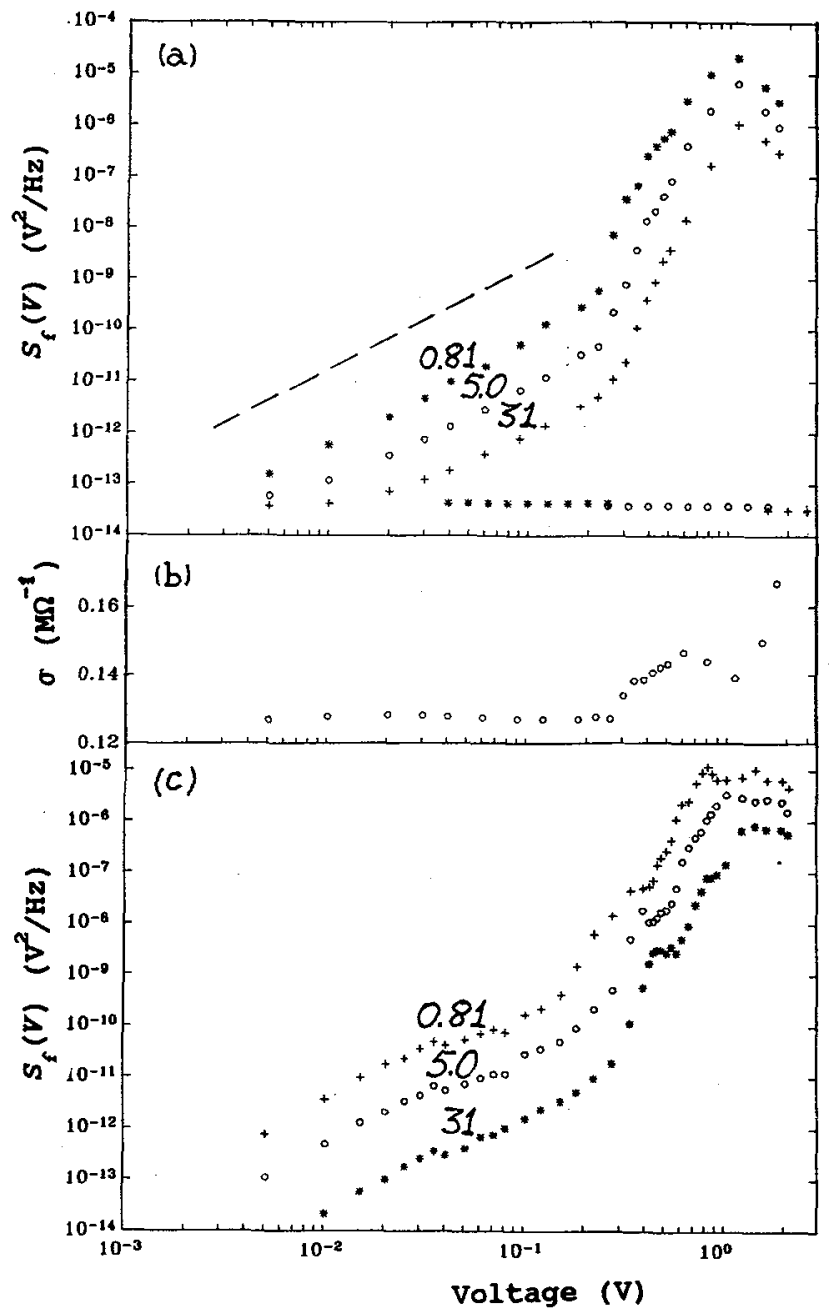

Fig. 1. a)-Voltage dependence of $S_{f}(V)$ at different $f$ (indicated in $\mathrm{Hz}$ ). 4-probe method. $\mathrm{T}=53 \mathrm{~K}$. Background $S_{f}(0)$ is shown at the right.

taneously.

$$
\text { b) }-\sigma(V) \text { measured simul- }
$$

method

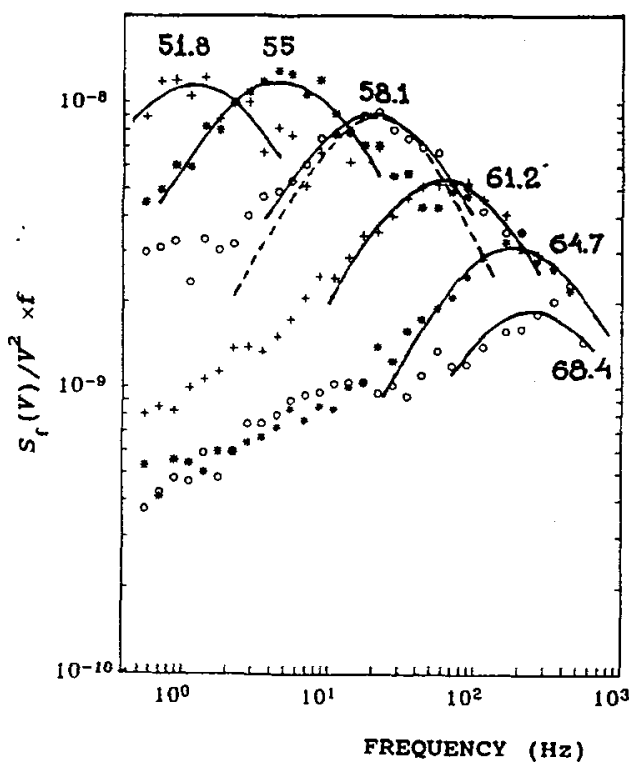

Fig. 2. The spectra multiplied by $f$ at different $T$ (indicated in K). 2-contact method. $\mathrm{V}=40 \mathrm{mV}$. The broken line corresponds to $S_{f}(V) \propto \tau_{0} /\left[1+\left(f / f_{0}\right)^{2}\right]$. The solid lines correspond to distribution of $\tau_{0}: \delta \ln \left(\tau_{0}\right)=0.65$.

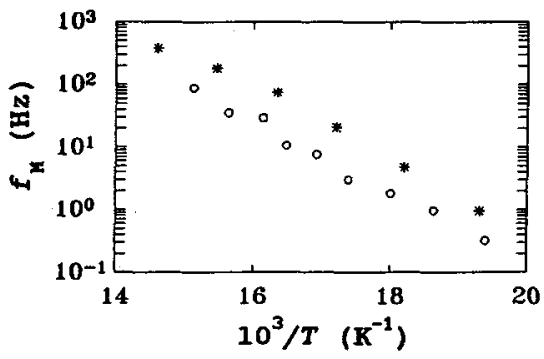

Fig. 3. The frequencies $f_{I}$, at which $S_{f}(V) \times f$ reaches maxima, vs. $10^{3} / T$. Data for 2 samples (* correspond to the spectra shown on fig.2).

frequencies (4-probe configuration). The abrupt growth of the noise around $V=200 \mathrm{mV}$ corresponds with the growth of conductivity $\sigma$ (fig. $1(b))$. The initial part of the curve $(V \leq 200 \mathrm{mV})$ indicates the voltage range at which $s_{f}(V)$ is proportional to $v^{\tilde{2}}$ (the dashed line). This means that the resistance fluctuations are nearly independent of the electric field applied. This result demonstrates existence of spontaneous resistance fluctuations of pinned CDW. 


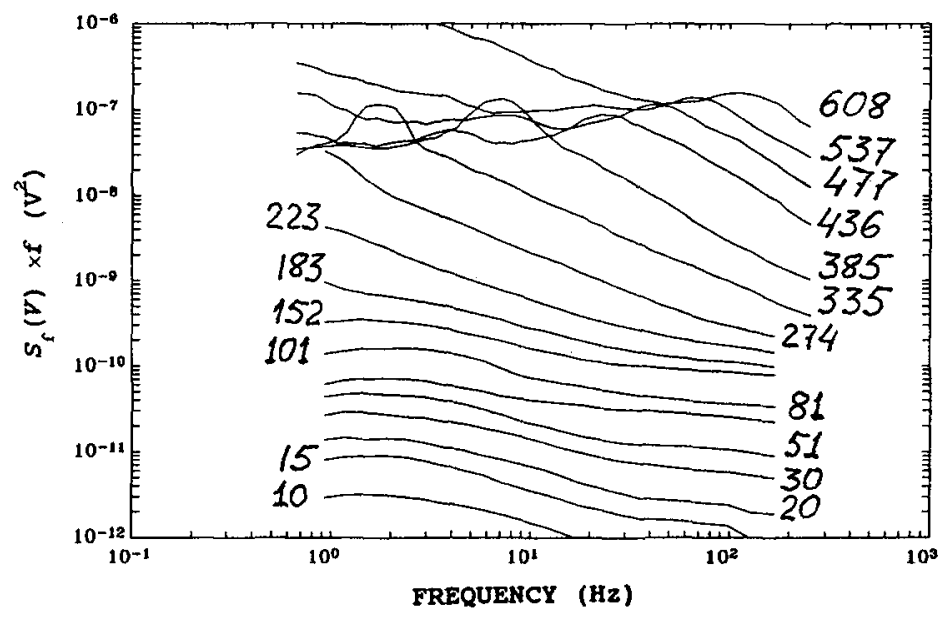

Fig. 4. $S_{f}(V) \times f$ vs. $f$ at different $V$ (indicated in mV). 2-probe method (see fig. 1c)

2-probe measurements give analogous curves for this sample (fig.l(c)), though influence of the electric field on fluctuations is observed at $V>40 \mathrm{mV}$, where the fluctuãtions are partly suppressed.

In all the samples we have observed growth of $S_{f}(V) / V^{2}$ at a given frequency by $2.5 \div 4$ orders of magnitude beginning from $T \sim 75 \mathrm{~K}$ down to $30 \div 40 \mathrm{~K}$, where $s_{f}(V) / v^{2}$ achieved $\sim 10^{-7} \mathrm{~Hz}^{-1}$ at $f=1 \mathrm{~Hz}$. 4and 2-contact curves demonstrate qualitatively analogous temperature dependencies of the noise. Thus, the noise is of volume origin. However, the 2-probe curves demonstrate more abrupt growth between 70 and $50 \mathrm{~K}$.

In the whole temperature range the dependence $S_{f}(V)$ on $f$, when measured by the 4-contact method, can be described as $1 / f^{\alpha}, \alpha \sim 1$. The 2-probe method gives, however, pronounced maxima in the frequency dependencies of $S_{f}(V) \times f$ between 70 and $50 \mathrm{k}$ (fig. 2), while the time domains of voltage demonstrate random switching between two levels. So, it was reasonable to approximate the spectra with a Lorentzian form (fig. 2, dashed line); better fit is achieved if we take into account a distribution of relaxation times, $\tau_{0}$ (fig. 2 , solid lines).

The frequencies $f_{M}$ corresponding to the maxima of $s_{f}(V) \times f$, yield the average values of $f_{0} \equiv 1 /\left(2 \pi \tau_{0}\right)$. It can be seen (fig. 2) that $f_{M}$ is temperature dependent. Fig. 3 shows temperature dependencies of $f_{M}$ for two samples, giving $\tau_{0}=\tau_{a} \exp (W / T)$, with $W \cong 1300 \mathrm{~K}$, and $\tau_{a} \sim 10^{-13} \mathrm{~s}$.

Though the 4-probe spectra did not demonstrate similar maxima, we found that the average slope, $\alpha$, of $\lg \left(S_{f}\right) \mathrm{vs}$. $\lg (1 / f)$ is changing from $\alpha<1$ to $\alpha>1$ with cooling the samples in the same range of $T$. From this we conclude that the average value of $\tau_{0}$ also decreases.

Fig. 4 shows the same measurements as fig.1(c) displayed as a set of $s_{f}(\delta V / V) \times f$ vs. $f$ at different $V$. At low voltages a maximum corresponding with fig.2 is seen. Its form looks voltage independent. No qualitative changes are observed until around $180 \mathrm{mV}$ extra noise appears at the lower frequencies. Then a maximum appeares at $f=f_{0}(V), f_{0}$ increasing with $V$. The same voltage dependence of $f_{0}$ can be obtained from the curves $s_{f}(\delta V / V) / V^{2}$ vs. $V$ at given $f$, if one marks $V$ corresponding to the first maximum (fig.1(c)). 4 - probe measurements yield similar growth of $f_{0}$ with $V$, though the maxima of $s_{f}(\delta V / V) \times f$ are less pronounced.

At low $T$ the screening of the defects in the volume is reduced due 
to freezing out of the quasiparticles. This can give rise to resistance fluctuations: growth of $1 / f-t y p e$ noise could be described by the semi-empirical Hooge relation [3] working well for semiconductors and metal films of different kind: $s_{f}(V) / V^{2} \times f=\gamma / N_{c}$, where $N_{c}$ is the total number of the free current carriers, and $\gamma \sim 2 \times 10^{-3}$. However, it gives the growth of $S_{f}(V) / V^{2} \propto R(T)$, which is slower than we have observed. Also, $S_{f}(V) / V^{2}$ appears at least 3 orders of magnitude lower than the experimental value. This discrepancy seems to indicate that the fluctuations have collective nature.

We suppose that the resistance fluctuations are caused by thermally-assisted motion of dislocations of the CDW. The role of dislocations, whose excitation energy (per one electron) is low in comparison with the over-gap excitations $[1,4]$, should increase at low $T$. The abrupt growth of noise below $75 \mathrm{~K}$ indicates that in this temperature range some extra defects develop in the volume of the CDW. Such defects are supposed to develop due to the reduction of screening of the CDW deformations $[5,6]$ providing strong pinning [2]. Growth of $E_{T}$ below $90 \mathrm{~K}$ and reduction of the dielectric constant [2] indicate decrease of the coherence volume. At the same time, $T>50 \mathrm{~K}$ appears high enough to provide thermal-assisted climb of dislocãtions between pinning centers, corresponding to the phase-slippage (PS) of the CDW. In this case, dislocation lines would cross CDW chains adding (or removing) 2 conducting electrons per chain, and giving rise to the fluctuations of conductivity [7]. The activation energy for $\tau_{0}(T), 1300 \mathrm{~K}$, characterizes the barrier for the climb of the dislocations. The underlying microscopic processes giving rise to this barrier remain unclear. Corresponding to the whole width of the Peierls gap, $W$ is still smaller, than the activation energy for the PS observed at higher $T$ [7]. We believe, that at $T<50 \mathrm{~K}$ the dislocations become immobile, forming the glassy state [2] and providing the relief for hopping conductivity.

The gradual growth of $f_{0}(V)$ at $V \sim V_{T}$ (fig. 4) indicates onset of the CDW current. The observed values of $f_{0}$ are too low to reveal the Fröhlich current directly. However, we found proportionality between $\delta \sigma$ and $\log \left(f_{0}\right)$, which corresponds to progressing PS activation with the deformation of the CDW. This result is in agreement with the logarithmic decay of metastable states in this temperature range [6].

one can see, that the relaxation times are better defined in the 2-contact configuration, which is evidently due to the synchronization of PS acts. In this case, nonuniform electric field near the current terminals, and, probably, the injection (or extinction) of free carriers by the terminals [4], affect the conditions of PS.

In conclusion, we have observed spontaneous resistance fluctuations at $T<80 \mathrm{~K}$ associated with thermally-assisted jumps of equilibrium dislocations of the CDW.

[1]. G. Grüner Rev. Mod. Phys. 60 (1988) 1129 ; P.Monceau 1985 in: Electronic Properties of Inorganic Quasi-one-dimensional compounds, Part 2. Ed. by P. Monceau. Dortrecht: D. Reidel Publ. Sump.

[2]. F. Ya. Nad' and P. Monceau Solid. State.Commun., in press

[3]. F. N. Hooge Phys. Lett. A 29 (1969) 139.

[4]. S. Brazovskii, S. Matveenko J. Phys. I France, 2 (1992) 409; ibid 2 (1992) 725

[5]. V. Ya. Pokrovskii and S. V. Zaitsev-Zotov Synthetic Metals, 29 (1989) F439; ibid 32 (1989) 321

[6]. S. V. Zaitsev-Zotov Synthetic Metals 41-43 (1991) 3923

[7]. D. V. Borodin, S. V. Zaitsev-Zotov and F. Ya. Nad' Zh. Eksp. Teor. Fiz. 93 (1987) 1394; [Sov. Phys. JETP 66 (1987) 793 